\title{
Molecular aggregation, solvato- and acidochromic properties of symmetrical ketocyanines
}

\author{
TAREK FAYED*, MOHAMMED EL-MORSI and MARWA EL-NAHASS \\ Department of Chemistry, Faculty of Science, Tanta University, 31527 Tanta, Egypt \\ e-mail: tfayed2003@yahoo.co.uk
}

MS received 8 August 2012; revised 27 December 2012; accepted 1 March 2013

\begin{abstract}
The absorption and fluorescence spectral characteristics of some symmetrical ketocyanine dyes have been investigated in different solvents of various polarities using steady-state absorption and emission spectroscopic techniques. The absorption spectral features are less sensitive to the solvent characteristics than the corresponding fluorescence spectra. A large excited state dipole moment parallel to the smaller ground state dipole moment was calculated. The large dipole moment change with the strongly solvatofluorochromic behaviour, as the solvent polarity increases, demonstrates the formation of an intramolecular charge-transfer (ICT) excited state, which is confirmed by theoretical calculation using semiempirical PM3 method. Also, the effect of dye concentration on the electronic absorption spectra for the investigated dyes has been studied in ethanol. In addition, the acidochromic behaviour of these dyes has been studied using sulphuric acid at different $H_{\mathrm{o}}$ values. A progressive red-shift in the absorption and emission spectra has been observed upon increasing the concentration of sulphuric acid with dramatic changes in colour. This suggests the design of potential optical sensors for probing acidity of the medium or water content in sulphuric acid. The calculated excited state protonation constants $\left(\mathrm{p} K_{\mathrm{a}}^{*}\right)$ are lower than $\mathrm{p} K_{\mathrm{a}}$ values calculated for the ground state, indicating that the investigated ketocyanines become more basic in the first excited singlet state.
\end{abstract}

Keywords. Ketocyanine dye; solvatochromic; intramolecular charge-transfer (ICT); molecular aggregation; acidochromic; protonation constant.

\section{Introduction}

Optical response of molecules showing an intramolecular charge-transfer (ICT) can serve as micropolarity and viscosity reporters probing local environments in simple solutions or complex biological systems. ${ }^{1-3}$ Ketocyanine dyes are good models for studying ICT, which have important implications in diverse fields such as photophysics, photochemistry, polymer physics, optics technology and laser physics. ${ }^{4}$ Many factors influence the spectral behaviour of dissolved molecules, especially the solvent polarity, temperature ${ }^{5}$ and hydrogenbond donor (HBD) or acceptor (HBA) capacity. ${ }^{6-8}$ Strong solvatochromic effects are generally observed for these dyes exhibiting large variations in their dipole moment during electronic transitions. These variations can differentially stabilize the ground and/or the excited state in polar and non-polar solvents. Hydrogen-bond formation also affects the molecular orbitals energy levels. Proton transfer reactions have been studied

*For correspondence extensively in the ground $\left(\mathrm{S}_{o}\right)$ and excited $\left(\mathrm{S}_{1}\right)$ states. $^{9}$ Acidochromic compounds are characterized by their ability to alternate between two distinct chemical forms having different absorption spectra in response to $\mathrm{pH}$ variations. Acidochromic dyes absorbing in the visible region are of particular importance, for instance, in sensor techniques, medical diagnostics and for potential sensitive probing. The charge density distribution in ketocyanines changes upon excitation of these molecules. This introduces large changes in their acidic and basic properties and thus leads to intermolecular solute-solvent proton transfer along the hydrogen bond in a tautomerization process (scheme 1).

The purpose of this study is to investigate the solvatochromic characteristics and photophysics of some symmetrical ketocyanines namely; 2,5-bis-[3(4-dimethylamino-phenyl)-allylidene-cyclopentanone (DPACP), 2,6-bis-[3-(4-dimethylamino-phenyl)-allylidene-cyclohexanone (DPACH) and 1,9-bis(4-(dimethylamino)phenyl)nona-1,3,6,8-tetraen-5-one (DPAE) in various homogeneous media by using the absorption and fluorescence spectroscopic techniques; supported by theoretical calculations. The acidochromic 


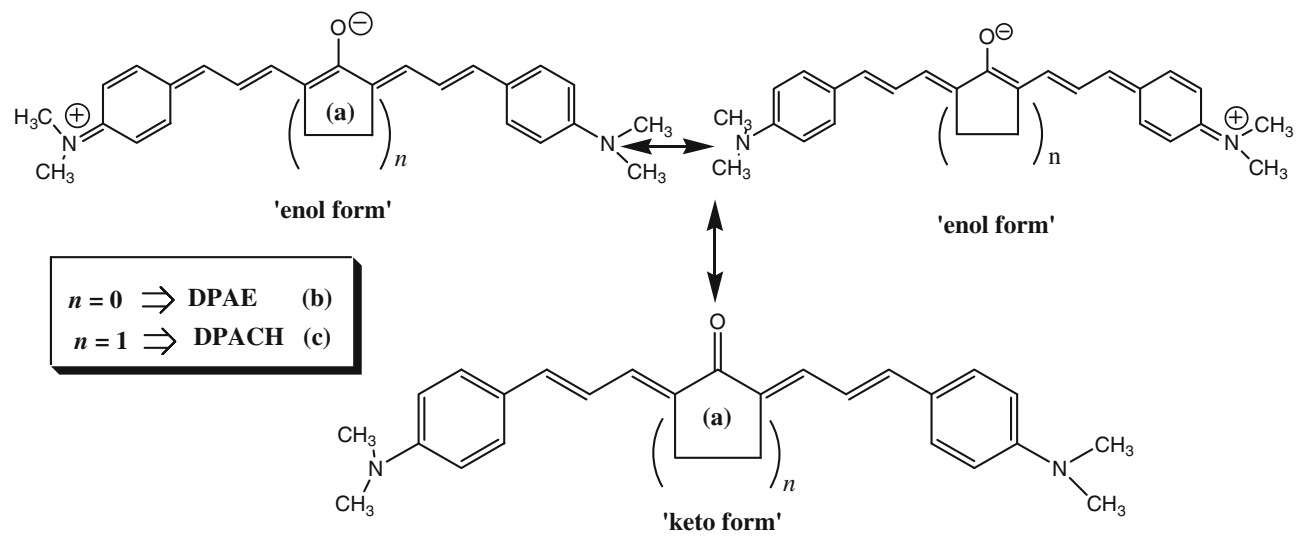

Scheme 1. Keto and charge-separated enol resonating structures of (a) DPACP, (b) DPACH and (c) DPAE.

behaviour of the investigated dyes has been also studied using Hammett's acidity function scale $\left(H_{\mathrm{o}}\right)$.

\section{Experimental details}

\subsection{Materials and methods}

The investigated ketocyanines; DPACP, DPACH and DPAE were synthesized and characterized following the procedures reported in a previous work. ${ }^{10}$ The used solvents such as; methanol (MeOH), methanol-d (MeOD), ethanol (EtOH) n-propanol $(\mathrm{PrOH})$, n-butanol $(\mathrm{BuOH})$, n-pentanol $(\mathrm{PenOH})$, acetonitrile (ACN), N,N-dimethylformamide (DMF), dichloromethane $\left(\mathrm{CH}_{2} \mathrm{Cl}_{2}\right)$, chloroform $\left(\mathrm{CHCl}_{3}\right)$, benzene (Benz) and n-heptane (Hep) were of spectroscopic grade from Aldrich, except glycerol (Gly) which was from Merck and used as received. All the solvents were found to be non-fluorescent in the scanned range.

Steady-state absorption and emission spectral measurements were carried out using a Shimadzu UV3101PC scanning spectrophotometer and a PerkinElmer LS 50B spectrofluorometer, respectively.

For studying the effect of hydrogen ion concentrations, Hammett's acidity function for sulphuric-water mixtures was used. ${ }^{11}$ Concentration of the investigated dyes was kept at $2 \times 10^{-5}$ moldm $^{-3}$ for all measurements.

Geometrical optimization along with electronic structure and dipole moment calculations of both the ground and excited states of the investigated ketocyanines have been performed with the help of ArgusLab 4.0 software $^{12}$ and free HyperChem 8.03 software. Geometrical optimization was obtained by PM3 Hamiltonian, while bond order, charge density and dipole moments were calculated by using ZINDO/S methods.

\section{Results and discussion}

\subsection{Theoretical calculations}

Geometrical and electronic structures of DPACP, DPACH and DPAE in the ground state have been elucidated using PM3 semiempirical calculations. The optimized molecular structures of the investigated dyes are shown in figure 1 . The full optimized geometry of DPACP and DPACH showed small deviation from planarity due to twisting of the dimethyl amino group at the two chromophore arms. In case of DPACP, the torsion angle for (C24, C26, N28 and C30) is $26.15^{\circ}$, and a similar value was calculated for $(\mathrm{C} 2, \mathrm{C} 3, \mathrm{~N} 6$ and $\mathrm{C} 8)$ in the same molecule. In the case of $\mathrm{DPACH}$, the torsion angle for (C3, C4, N7 and C9) and (C24, C27, $\mathrm{N} 28$ and $\mathrm{C} 29$ ) is $29.55^{\circ}$. However, in DPAE, the calculations showed stable structure with a minimum energy at torsion angles equal to $0^{\circ}$ or $180^{\circ}$, suggesting a planar structure. The bond orders and charge densities of the investigated dyes in the ground and excited states have been calculated using ZINDO/S semiempirical calculations and summarized in table 1. As can be seen, the values of bond order calculated for the excited singlet state, show an inverted alternation where the bonds characterized by single bond characters in the ground state acquire double bond characters in the excited state and vice versa. These changes indicate a strong coupling between the electron donor two dimethyl amino groups, at both arms, and the electron acceptor ketogroup as an, via the butadiene bridges. Further, confirmation for this fact comes from charge density calculations. The data show that the charge density at the oxygen atom of carbonyl group increases from $-0.6064,-0.605$ and $-0.6202 \mathrm{e}$ to $-0.7062,-0.6986$ and -0.7058 e for DPACP, DPACH and DPAE, respectively, while that on the nitrogen atom of the dimethylamino group, decreases from $-0.3719,-0.3722$ and 

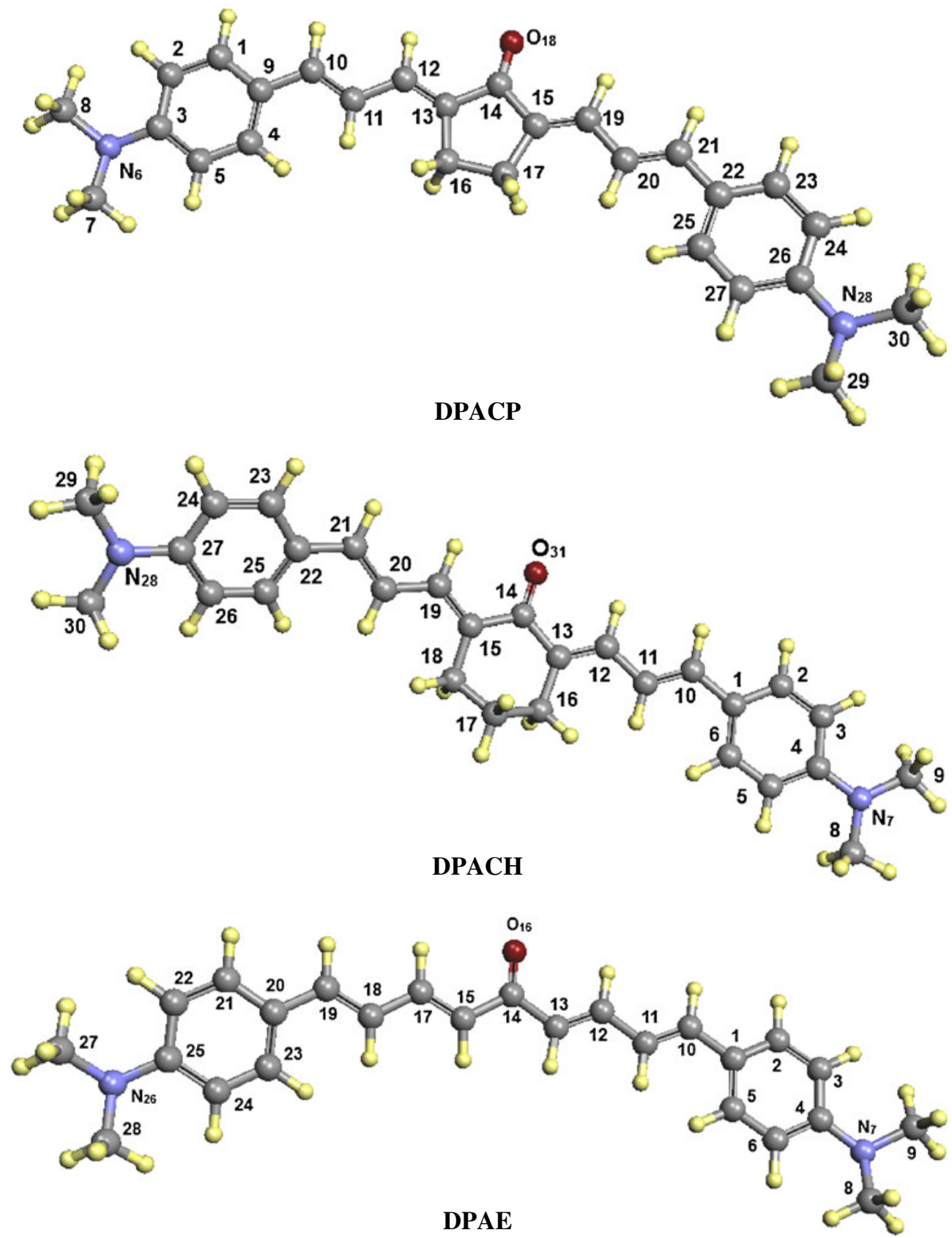

Figure 1. The optimized geometry of ground state of the investigated ketocyanines using PM3 calculations.

$-0.3902 \mathrm{e}$ to $-0.3547,-0.3553$ and $-0.3555 \mathrm{e}$ upon excitation. The HOMO and LUMO of the investigated ketocyanines are depicted in figure 2 which shows that the HOMO of these dyes is characterized by large electron density on the nitrogen atoms relative to carbonyl oxygen. On the other hand, the electron density on carbonyl oxygen becomes relatively larger in LUMO. Thus, the HOMO-LUMO transition within the dye is associated with an intramolecular charge-transfer (ICT) character. Semiempirical calculations using ZINDO/S methods were also extended to provide the ground and excited singlet state dipole moments in the gas phase (table 2). Pronounced increase in the dipole moments of the excited state of the investigated ketocyanines was found confirming ICT transitions.

\subsection{Solvatochromic behaviour of the investigated ketocyanines}

3.2a Electronic absorption spectra: Absorption spectra of DPACP, DPACH and DPAE were recorded in 
Tarek Fayed et al.

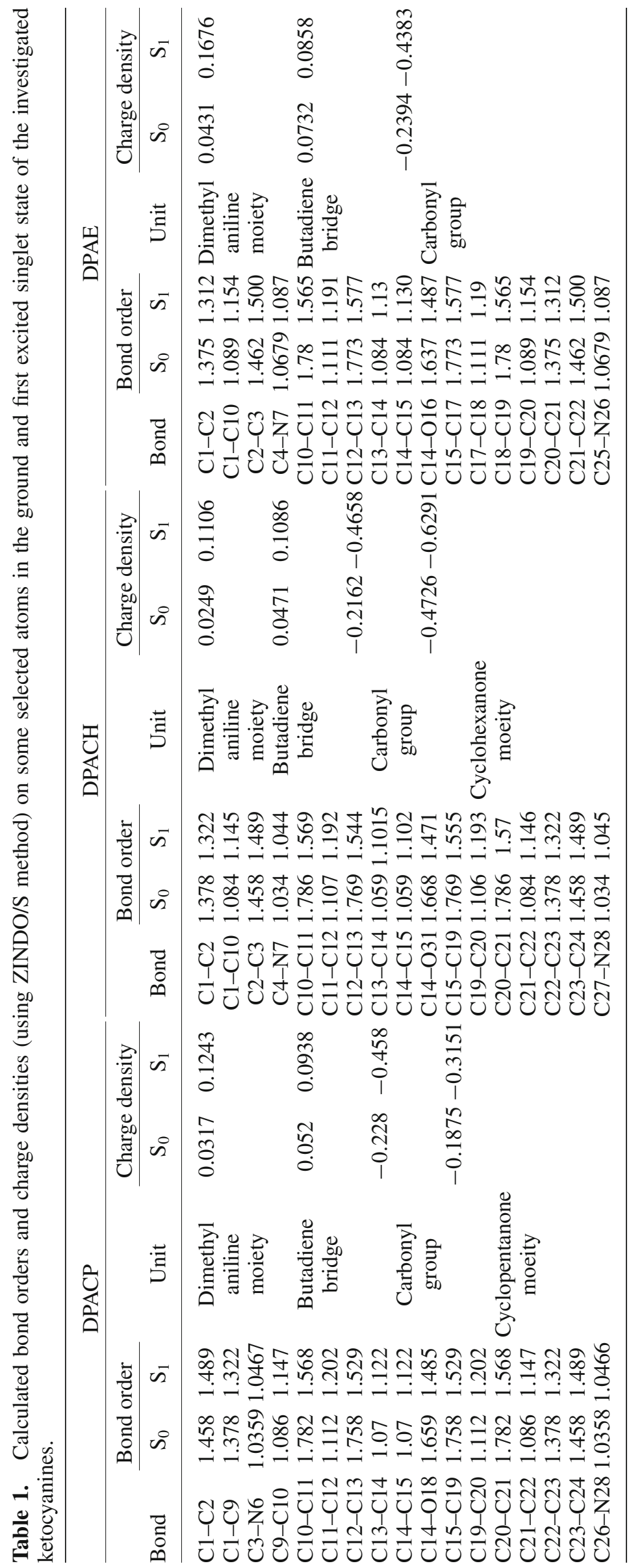




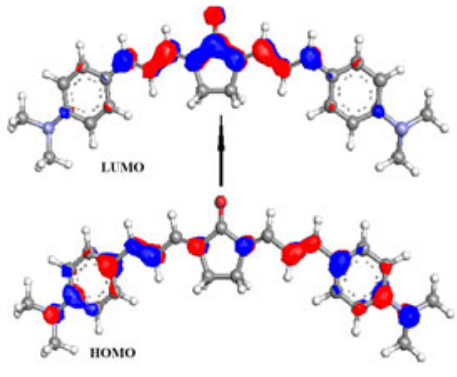

DPACP

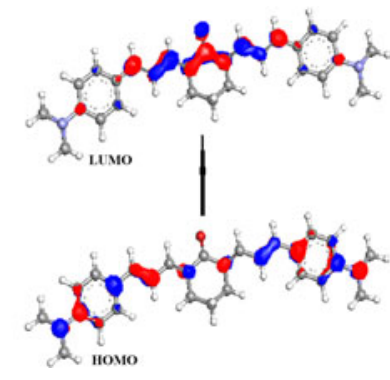

DPACH

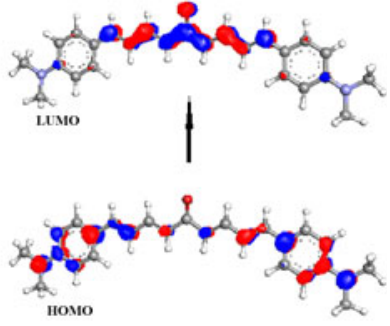

DPAE

Figure 2. HOMO-LUMO of the investigated ketocyanines.

several solvents of different polarity and proton donating ability at room temperature. Figure 3(a) shows normalized absorption spectra of the investigated ketocyanines in $\mathrm{n}$-heptane and $\mathrm{MeOH}$ as examples of non-polar and polar solvents, respectively. Electronic absorption properties of DPACP, DPACH and DPAE depend on the nature of the used solvent. Solutions vary in colour from faint yellow to purple for DPACP and $\mathrm{DPACH}$, and from faint yellow to light blue for DPAE, as the solvent is changed from n-heptane to proton donor alcohols; thus, relating the sensing ability of these ketocyanines to the polarity and hydrogen bonding characters of the solvent. The observed solvatochromic behaviour is due to the presence of such ketocyanines in two tautomeric forms (keto and charged enol forms, scheme 1). The extent of contribution of both tautomers in solution is governed by the nature and polarity of the used solvent. The less polar keto form contributes mainly in non- and less polar solvents, so, the faint yellow colour appears. In contrast, the highly polar enol form predominates in polar and strong hydrogen bonding donor solvents, thus, imparting deep colours to the solutions. The long-wavelength electronic transitions of the investigated dyes are characterized by higher molar absorbance $\left(\varepsilon_{\max }\right.$ ranges from $1.32 \times 10^{4}$ to $\left.1.5 \times 10^{5} \mathrm{~L} \mathrm{~mol}^{-1} \mathrm{~cm}^{-1}\right)$. Also, the band maximum undergoes pronounced red-shifts with increase in the solvent polarity (ca. 45, 34 and $25 \mathrm{~nm}$ for DPACH, DPAE and DPACP, on going from Hep to DMF, respectively) (table 3). The observed solvent sensitivity is intelligible in terms of the $\pi \rightarrow \pi^{*}$ transition with ICT from the dimethyl amino group to the carbonyl oxygen.

Fluorescence quantum yields $\left(\Phi_{f}\right)$ of the investigated ketocyanines have also been measured in different solvents, and summarized in table 3 . The $\Phi_{f}$ values are highly sensitive to solvent polarity and acidity. Generally, the values decrease steadily as the hydrogen bond donating ability of the solvents increases indicating that hydrogen bonding interactions strongly control the decay of excited molecules. The $\Phi_{f}$ value of DPACH and DPAE decreases from 0.09 and 0.18 in nheptane to 0.031 and 0.005 in $\mathrm{EtOH}$, respectively. This is probably due to the coupling between the close-lying $\mathrm{n}-\pi^{*}$ and $\pi-\pi^{*}$ states, which increases the possibility of non-radiative transition to deactivate the excited state. The highly polar excited state becomes more stabilized, thus decreasing the energy gap between the $\pi-$ $\pi^{*}$ and $\mathrm{n}-\pi^{*}$ states. This leads to the observed nonradiative losses of the excited state energy. This is confirmed by the finding that the $\Phi_{f}$ values in EtOH as a protic solvent are less than that of ACN. In contrast, the $\Phi_{f}$ value of DPACP increases from 0.039 in Hep to 0.066 in EtOH. This may be due to weakening of the coupling between ${ }^{1}\left(\mathrm{n}-\pi^{*}\right)$ and ${ }^{1}\left(\pi-\pi^{*}\right)$ states in EtOH. The ${ }^{1}\left(\pi-\pi^{*}\right)$ state is lowered and the ${ }^{1}\left(\mathrm{n}-\pi^{*}\right)$ state is elevated in $\mathrm{EtOH}$, increasing the energy gap between the states.

Table 2. Slopes $\left(\mathrm{m}_{1}\right.$ and $\left.\mathrm{m}_{2}\right)$ and the ground and excited state dipole moments calculated using experimental and theoretical methods.

\begin{tabular}{lccccccc}
\hline \multirow{2}{*}{ Dye } & \multicolumn{4}{c}{ Experimental } & & \multicolumn{2}{c}{ Theoretical } \\
\cline { 2 - 4 } & $\mathrm{m}_{1}\left(\mathrm{~cm}^{-1}\right)$ & $\mathrm{m}_{2}\left(\mathrm{~cm}^{-1}\right)$ & $\mu_{g}(\mathrm{D})$ & $\mu_{e}(\mathrm{D})$ & & $\mu_{g}(\mathrm{D})$ & $\mu_{e}(\mathrm{D})$ \\
\hline DPACP & 2126 & 4116 & 6.01 & 18.9 & & 6.97 & 14.77 \\
DPACH & 1944 & 4579 & 7.9 & 19.6 & & 6.92 & 18.28 \\
DPAE & 1824 & 3475 & 5.6 & 17.9 & & 6.41 & 14.52 \\
\hline
\end{tabular}



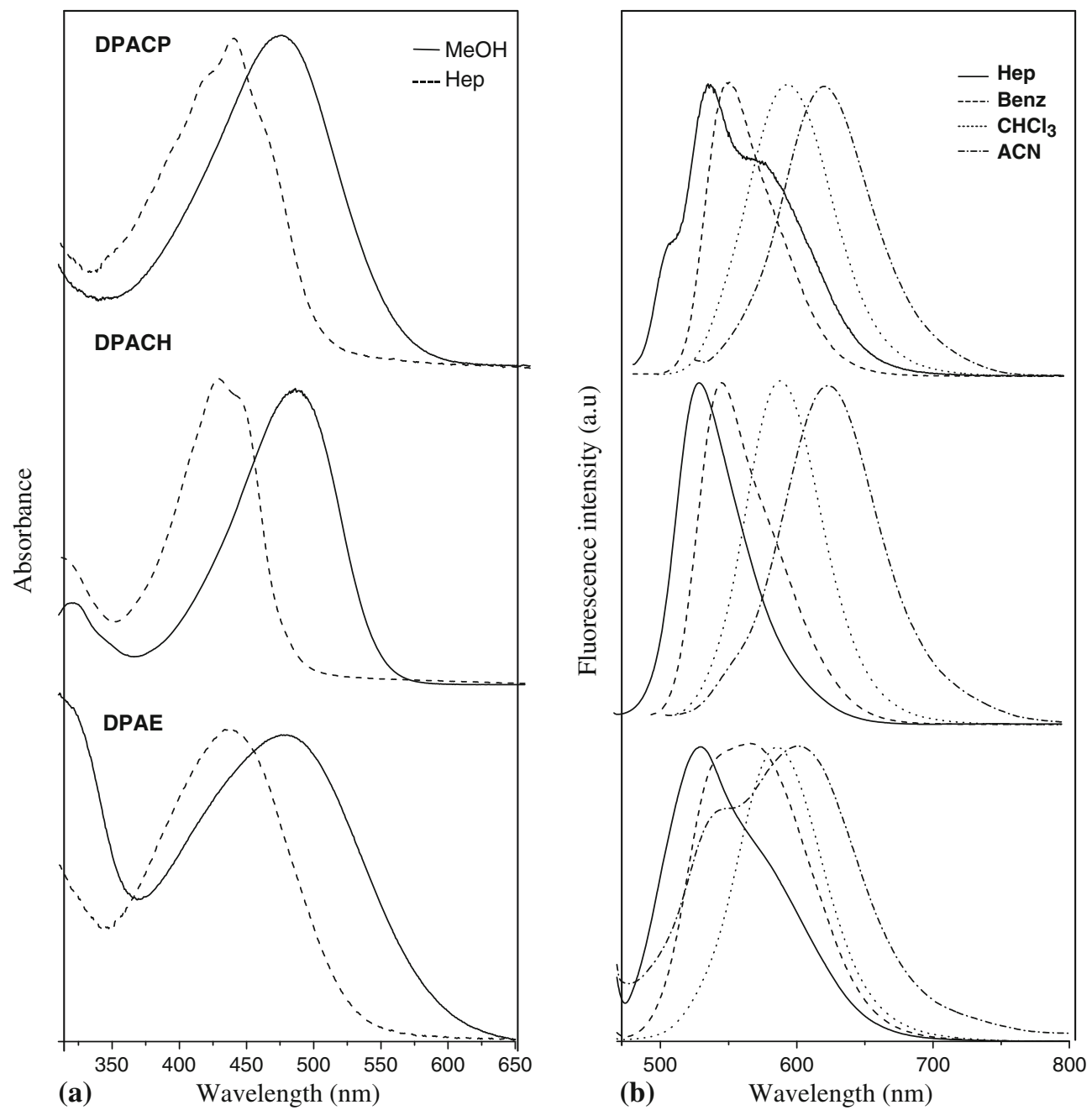

Figure 3. Normalized (a) absorption and (b) emission spectra of the investigated ketocyanines in the mentioned solvents represents inside the figure.

Table 3. Spectral data of the investigated dyes in various solvents at $25^{\circ} \mathrm{C}$.

\begin{tabular}{|c|c|c|c|c|c|c|c|c|c|}
\hline \multirow[b]{2}{*}{ Solvent } & \multicolumn{3}{|c|}{ DPACP } & \multicolumn{3}{|c|}{ DPACH } & \multicolumn{3}{|c|}{ DPAE } \\
\hline & $\lambda_{\max }^{a}(\mathrm{~nm})$ & $\lambda_{\max }^{f}(\mathrm{~nm})$ & $\Phi_{f}$ & $\lambda_{\max }^{a}(\mathrm{~nm})$ & $\lambda_{\max }^{f}(\mathrm{~nm})$ & $\Phi_{f}$ & $\lambda_{\max }^{a}(\mathrm{~nm})$ & $\lambda_{\max }^{f}(\mathrm{~nm})$ & $\Phi_{f}$ \\
\hline Hep & 480 & $497,533,576$ & 0.039 & 436 & 519 & 0.09 & 436 & 528 & 0.18 \\
\hline Benz & 492 & 550 & 0.056 & 461 & 534 & 0.15 & 459 & 557 & 0.32 \\
\hline $\mathrm{CHCl}_{3}$ & 503 & 612 & 0.43 & 482 & 580 & 0.73 & 472 & 596 & 0.42 \\
\hline $\mathrm{CH}_{2} \mathrm{Cl}_{2}$ & 504 & 618 & 0.54 & 497 & 600 & 0.75 & 471 & 602 & 0.38 \\
\hline DMF & 505 & 632 & 0.23 & 481 & 612 & 0.19 & 470 & 603 & 0.46 \\
\hline $\mathrm{ACN}$ & 495 & 631 & 0.162 & 476 & 620 & 0.176 & 459 & 542,602 & 0.35 \\
\hline n-PenOH & 521 & 637 & 0.106 & 492 & 537,626 & 0.17 & 481 & 535,599 & 0.16 \\
\hline n-BuOH & 519 & 636 & 0.093 & 492 & 538,627 & 0.072 & 475 & 534,602 & 0.12 \\
\hline $\mathrm{n}-\mathrm{PrOH}$ & 512 & 632 & 0.088 & 488 & 540,627 & 0.06 & 473 & 535,602 & 0.09 \\
\hline $\mathrm{EtOH}$ & 519 & 623 & 0.066 & 490 & 539,625 & 0.031 & 480 & 530,600 & 0.005 \\
\hline MeOD & 521 & 648 & 0.025 & 493 & 540,623 & 0.021 & 480 & 529,599 & 0.003 \\
\hline $\mathrm{MeOH}$ & 523 & 621 & 0.016 & 491 & 539,624 & 0.018 & 476 & 529,591 & 0.003 \\
\hline Glycerol & 436 & 603 & 0.18 & 421 & 491 & 0.605 & 418 & 584 & 0.39 \\
\hline $\mathrm{H}_{2} \mathrm{O}$ & 427 & 587 & 0.0154 & 414 & 601 & 0.015 & 409 & 574 & 0.014 \\
\hline
\end{tabular}




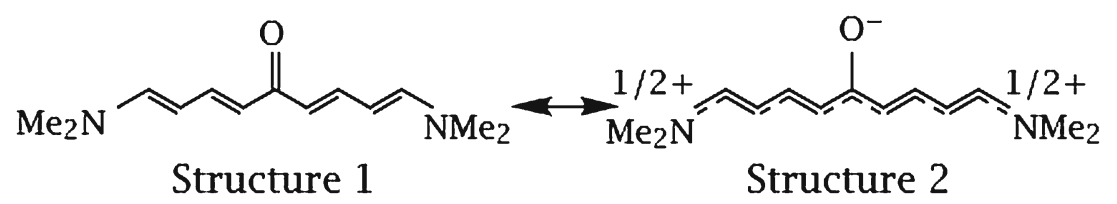

Scheme 2. $\pi$-Electronic structure of ketocyanine dyes.

To highlight the effect of the molecular structure on the under investigation spectral behaviour, the absorption and emission spectra of the symmetrical ketocyanines (DPACP, DPACH and DPAE, with two amino polyenic fragments of $\mathrm{D}-\pi-\mathrm{A}-\pi-\mathrm{D}$ configuration) have been compared with an unsymmetrical ketocyanine dye (with one amino polyenic fragments of $D-\pi-$ A configuration, abbreviated as DMAPT and reported in one of our earlier studies ${ }^{13}$ in $\mathrm{MeOH}$ as a representative solvent. The absorption and fluorescence bands of symmetric DPACP, DPACH and DPAE are red-shifted compared to unsymmetric DMAPT. This red-shift follows the order DMAPT $<$ DPAE $<$ DPACH $<$ DPACP, which can be explained on the basis of extension of the conjugation via the two aminopolyenic chromophores. The $\pi$-electronic structure of a ketocyanine dye can be represented as intermediate between two limiting resonance structures (structures 1 and 2, scheme 2). Structure 1 is characterized by alternating polyenic bonds and absence of charge separation; structure 2 has complete $\pi$-electron conjugation along the whole polyenic chain and separate charges. As an example, replacement of tetralone moiety in unsymmetric DMAPT by dimethylamino-allydiene cyclohexanone fragment in symmetric DPACH (i.e., elongation of the area of electron delocalization) enhances the resonance delocalization of the $\pi$-electrons and leads to progressive shift in the absorption and emission maxima. Quantitatively, the absorption and emission spectra are red-shifted from 445 and $623 \mathrm{~nm}$ to 491 and $624 \mathrm{~nm}$ for DMAPT and DPACH, respectively in $\mathrm{MeOH}$. Also, the fluorescence quantum yield $\Phi_{f}$ varies strongly with the molecular structure. The $\Phi_{f}$ values for unsymmetric DMAPT in $\mathrm{MeOH}$ is much higher than that for the corresponding symmetric DPACP, DPACH and DPAE, indicating quenching of the emitting state for symmetric dye through the enhanced ICT interaction (the $\Phi_{f}$ values of DMAPT, DPACP, DPACH and DPAE in $\mathrm{MeOH}$ are $0.229,0.016,0.018$ and 0.003 , respectively). On the other hand, from charge density calculations, it was found that replacement of tetralone moiety in DMAPT by dimethylamino-allydiene cyclohexanone fragment in DPACH perturbs the charge distribution over the span of excited molecule. This can be seen from the pronounced decreasing in the charge density at three subunits carbonyl group, butadiene bridge and dimethyl aniline moiety of DPACH by $0.0061,0.1299$ and $0.0868 \mathrm{e}$, respectively, therefore, the carbonyl group in DPACH is more electronwithdrawing compared to that of DMAPT. One of the effects of increasing the length of the chain is the increasing of dipole moment change upon excitation. As a representative example, the excited dipole moment of DPACH in the gas phase was 18.28 , while the corresponding one for DMAPT was 12.72 , confirming that intramolecular charge transfer within DPACH is more efficient than that of DMAPT. This could confirm the great bathochromic shift of absorption maximum of DPACH relative to that of DMAPT.

3.2b Molecular aggregation of the investigated ketocyanines in ethanolic solutions: A cursory glance at the absorption spectra of the investigated ketocyanines in different solvents reflects significant variations in the shape and peak position on going from EtOH to pure water. Precisely, the broad absorption band of DPACP, DPACH and DPAE in EtOH that appears at 519, 490 and $480 \mathrm{~nm}$, respectively, is replaced by a narrow band at 427,414 and $409 \mathrm{~nm}$ in water, with a broad tail around 550, 565 and $579 \mathrm{~nm}$. Based on the shape of the absorption band, the large hypsochromic shift and narrowing of the band in water, is due to formation of $\mathrm{H}$-aggregates ${ }^{14}$ while the broad tail was attributed to absorption of monomers.

The electronic absorption spectra of ketocyanine solutions with different concentrations have been studied in $\mathrm{EtOH}$, figure 4a. The spectra display two absorption peaks, the first peak at 309, 315 and $307 \mathrm{~nm}$ is of low intensity, and the second peak is of higher intensity and appears at 516, 494 and $484 \mathrm{~nm}$, for DPACP, DPACH and DPAE, respectively. As the concentration of DPACP, DPACH and DPAE increases from 6 $\times 10^{-6}$ to $2.6 \times 10^{-4} \mathrm{M}$ the molar absorptivity of the short-wavelength band increases at the expense of that of the long-wavelength band. Figure $4 \mathrm{~b}$ shows the molar absorption spectra of the investigated ketocyanines in EtOH as a function of dye concentration. The spectra of DPACP, DPACH and DPAE pass through a well-defined isosbestic point at 392, 404 and $397 \mathrm{~nm}$, 

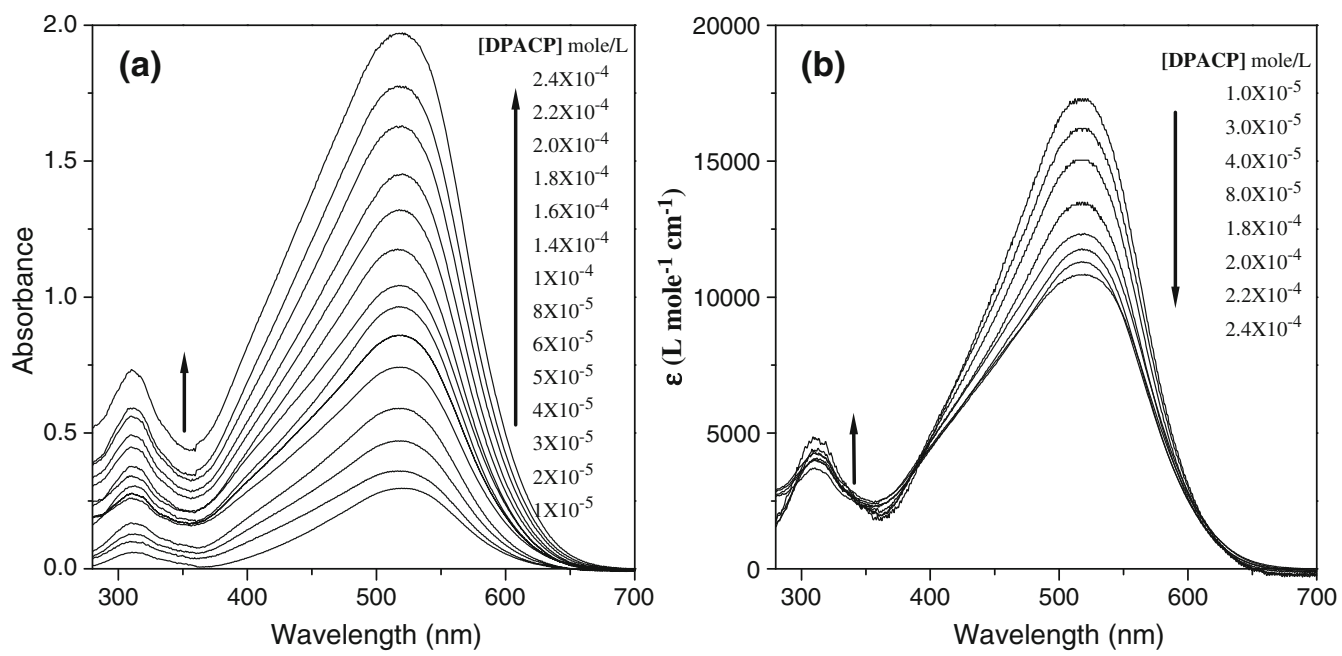

Figure 4. Absorption spectra of DPACP in EtOH at different concentrations.

for DPACP, DPACH and DPAE, respectively, indicating the presence of an equilibrium between two different species namely; monomers (M) and probably dimers (D) in solution. In addition, the spectra show a hypochromic effect for the long-wavelength charge transfer band and concomitantly hyperchromic effect for the shorter-wavelength band. This behaviour is characteristic for molecular aggregation and formation of dimers or $\mathrm{H}$-aggregates.

In order to obtain information about the aggregation process, whether dimers or higher aggregates are formed, the aggregation number $(n)$ and the aggregation constant $\left(K_{\mathrm{d}}\right)$ of the investigated dyes were determined using the following equation: ${ }^{15}$

$$
\log \left(\left(1-f_{m}\right) C\right)=n \log \left(f_{m} C\right)+\log K_{d}+\log n,
$$

where $f_{m}$ indicates the ratio of the monomer with the overall concentration, with limiting values of zero (pure dimer) and unity (pure monomer) and $C$ is the overall concentration of the dye.

Having obtained a set of spectra at different concentrations, the values of $\left(1-f_{m}\right)$ for the investigated dyes can be calculated by using the following equation:

$$
1-f_{m}=\frac{\left(\varepsilon_{\lambda_{1}} / \varepsilon_{\lambda_{2}}\right)_{\max }-\left(\varepsilon_{\lambda_{1}} / \varepsilon_{\lambda_{2}}\right)}{\left(\varepsilon_{\lambda_{1}} / \varepsilon_{\lambda_{2}}\right)_{\max }-\left(\varepsilon_{\lambda_{1}} / \varepsilon_{\lambda_{2}}\right)_{\min }},
$$

where $\lambda_{1}$ is the wavelength at which the change in $\varepsilon$ value for the conversion of the monomer to the dimer was highest (there is a monomer absorption peak, and its intensity considerably decreases by increasing the concentration of the solution) and $\lambda_{2}$ represents the isosbestic point. The division of $\varepsilon_{\lambda_{1}}$ by that of the isosbestic point $\varepsilon_{\lambda_{2}}$ reduces the effects of nonsystematic errors in the values of $C$ of the samples.
Maximum and minimum values of $\varepsilon_{\lambda_{1}} / \varepsilon_{\lambda_{2}}$ are obtained by plotting the relation between $\varepsilon_{\lambda_{1}} / \varepsilon_{\lambda_{2}}$ and $\log C$ at different dye concentrations. The obtained limiting values of $\left(\varepsilon_{\lambda_{1}} / \varepsilon_{\lambda_{2}}\right)_{\max }$ are 3.44, 4.37 and 2.69 for DPACP, DPACH and DPAE, respectively, while $\left(\varepsilon_{\lambda_{1}} / \varepsilon_{\lambda_{2}}\right)_{\text {min }}$ values are $2.76,2.78$ and 2.06 , respectively. The $f_{m}$ values were calculated using these values. Plots of $\log \left(\left(1-f_{m}\right) C\right)$ versus $\log \left(f_{m} C\right)$ for the investigated ketocyanines are linear. The aggregation number $(n)$ has been determined from the slope and the aggregation constant $K_{d}$ was determined from the intercept (table 4). The values obtained for aggregation degree of DPACP, DPACH and DPAE are 2.15, 2.01 and 2.22 ( $n$ $\approx 2$ ), confirming that the aggregation process is due to dimer formation and the possibility of higher aggregates was ruled out.

3.2c Fluorescence characteristics of ketocyanines in different solvents: Figure 3(b) displays emission spectra of DPACP, DPACH and DPAE in some selected solvents, and the corresponding fluorescence data are collected (table 3). The band maximum is shifted dramatically to red with increase in solvent polarity and HBD ability indicating an increase in the dipole moment of the excited state.

Table 4. Aggregation constant $\left(\mathrm{K}_{d}, \mathrm{~L} / \mathrm{mol}\right)$, aggregation number $(n)$, and the correlation coefficient $(\mathrm{R})$ for the investigated ketocyanines.

\begin{tabular}{lccc}
\hline Dye & $\mathrm{K}_{d} \mathrm{~L} / \mathrm{mol}$ & $n$ & $\mathrm{R}$ \\
\hline DPACP & 1281 & 2.2 & 0.99 \\
DPACH & 948 & 2.0 & 0.996 \\
DPAE & 1636 & 2.2 & 0.97 \\
\hline
\end{tabular}


Fluorescence spectra of DPACP and DPAE appears as broad and structureless bands in the used solvents, while in non-polar solvents (such as Hep), the fluorescence spectra are structured. As seen, the emission spectrum of DPACP and DPAE fluoresce with vibronic structure at $497 \mathrm{~nm}$ and $533 \mathrm{~nm}$ in addition to a shoulder at $576 \mathrm{~nm}$, and at $530 \mathrm{~nm}$ and $570 \mathrm{~nm}$, respectively. Similarly, the fluorescence spectrum of DPAE appears as a broad and structured band in benzene. This was attributed to the formation of two emission bands. The origin of these structured bands may be due to the participation of two different excited states; the first state is locally excited singlet state (LE) and the second state may be ICT.

In contrast, the emission of DPACH does not exhibit fine structure. Also, the fluorescence spectrum of DPAE in the aprotic solvent ACN exhibits dual emission which could be attributed to the presence of LE and ICT states. The ICT from the donor to the acceptor produces a low lying anomalous CT state in aprotic polar solvents as reported for structurally related dyes. ${ }^{16}$ This ICT state leads to the new longer wavelength fluorescence band (around $602 \mathrm{~nm}$ ) in addition to emission, from the LE state at $542 \mathrm{~nm}$.

Alcoholic solutions of DPACH and DPAE exhibit dual emission which may arise from population of a twisted ICT state in addition to the normal ICT state. In protic solvents, the carbonyl group of the dye could be hydrogen bonded to the proton donor solvent molecules and the strength of the hydrogen bond becomes stronger in the excited state. This is due to increase in charge density on the oxygen atom of the carbonyl group upon excitation as a result of the enhanced ICT interaction. The emission of these dyes in a protic solvent takes place from a different emitting species, presumably a mesomeric enol. ${ }^{17}$

The difference between the excited singlet and ground dipole moments ( $\mu_{e}$ and $\mu_{g}$, respectively) can be determined using Bakhshiev's (eq. 3) ${ }^{18}$ and KawskiChamma-Viallet's (eq. 4). ${ }^{19}$

$$
\begin{gathered}
v_{\mathrm{abs}}-v_{\mathrm{em}}=m_{1} f(\varepsilon, n)+\mathrm{constant} \\
v_{\mathrm{abs}}+v_{\mathrm{em}}=-m_{2}[f(\varepsilon, n)+2 g(n)]+\mathrm{constant},
\end{gathered}
$$

where $v_{\text {abs }}$ and $v_{\text {em }}$ are the absorption and fluorescence band shift in solvents of varying permittivity $(\varepsilon)$ and refractive index $(n) . f(\varepsilon, n)=\frac{2 n^{2}+1}{n^{2}+2}\left[\frac{\varepsilon-1}{\varepsilon+2}-\frac{n^{2}-1}{n^{2}+2}\right]$ is the solvent polarity parameter and $g(n)=\frac{3}{2}\left[\frac{n^{4}-1}{\left(n^{2}+2\right)^{2}}\right]$ with $m_{1}=\frac{2\left(\mu_{e}-\mu_{g}\right)^{2}}{h c a^{3}}$ and $m_{2}=\frac{2\left(\mu_{e}^{2}-\mu_{g}^{2}\right)}{h c a^{3}}$, where, $h$ is Planck's constant and $c$ is the velocity of light in vacuum. The parameters $m_{1}$ and $m_{2}$ can be determined from the plots of absorption and fluorescence band shifts $\left(v_{\mathrm{abs}}-v_{\mathrm{em}}\right.$ and $\left.v_{\mathrm{abs}}+v_{\mathrm{em}}\right)$ with the solvent polarity parameters according to the above equations. If the ground and excited states are parallel, the following expressions are obtained on the basis of above equations. ${ }^{20}$

$$
\begin{aligned}
& \mu_{g}=\frac{m_{2}-m_{1}}{2}\left[\frac{h c a^{3}}{2 m_{1}}\right]^{\frac{1}{2}} \\
& \mu_{e}=\frac{m_{2}+m_{1}}{2}\left[\frac{h c a^{3}}{2 m_{1}}\right]^{\frac{1}{2}} .
\end{aligned}
$$

The cavity radius was taken as $40 \%$ from the distance between the two farthest atoms in the direction of charge separation within the molecule. ${ }^{21}$ The values were estimated following geometry optimization of DPACP, DPACH and DPAE and were to be 9.2, 8.9 and $9.4 \AA$, respectively. The dipole moment values are collected in table 2 together with the slopes of the corresponding solvatochromic shifts. The estimated ground state dipole moments for DPACP, DPACH, and DPAE are 6.01, 7.9 and 5.6 D and that of the excited state are 18.9, 19.6 and 17.9 D. This indicates the existence of a more relaxed excited state, due to ICT favoured by the cooperative effects of the dimethyl aniline moieties as donors and the carbonyl group as an acceptor and suggests that the present dyes can serve as good candidate components of non-linear optical materials. The estimated values are in good agreement with those obtained theoretically using ZINDO/S methods in the gas phase, (table 2).

\subsection{Acidochromic and prototropic equilibria of the investigated ketocyanines}

Acidochromic behaviour of the investigated ketocyanines was explored by studying the electronic absorption and emission spectra of $2 \times 10^{-5} \mathrm{~mol} \mathrm{dm}^{-3}$ of DPACP, DPACH and DPAE at different hydrogen ion concentrations $\left(H_{\mathrm{o}}\right)$ using sulphuric acid, figure 5(a) and (b). The spectral data are compiled in table 5 Absorption spectra of the investigated ketocyanines changes strongly upon increasing the concentration of sulphuric acid $\left(H_{\mathrm{o}}\right.$ ranges from -0.31 to -8.91$)$ with a dramatic change in colour, inset of figure 5(a). As an example, the DPACP solution changes from yellow to violet passing through orange and pink colours as the concentration of sulphuric acid was increased. Actually, the yellow colour is recovered upon the addition of sodium hydroxide solution which is evidence for the 

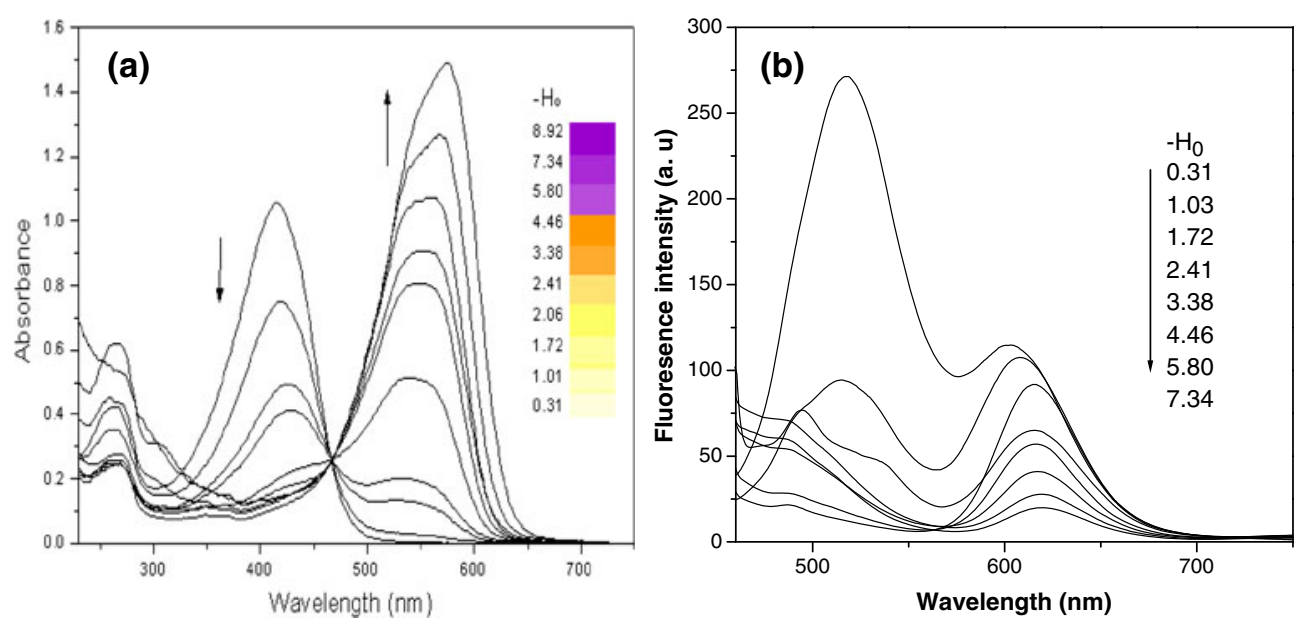

Figure 5. Variation of (a) absorption and (b) emission spectra of DPACP, with sulphuric acid concentration.

reversibility of the prototropic equilibrium that takes place. The investigated DPACP, DPACH and DPAE are neutral $\left(\lambda_{\max }=427,414\right.$ and $409 \mathrm{~nm}$, respectively). However, on adding different concentrations of sulphuric acid to the dye solutions $\left(H_{\mathrm{o}}\right.$ ranges from -0.31 to -2.41 ) absorption bands appear at 415,397 and $373 \mathrm{~nm}$ for DPACP, DPACH and DPAE, respectively. This short-wavelength absorption band is a characteristic for protonation of the $-\mathrm{NMe}_{2}$ group and formation of monocation I. The intensity of this band decreases at higher concentrations of sulphuric acid $\left(H_{\mathrm{o}}\right.$ ranges from -2.85 to -8.92 ) and new absorption bands develop at 574,554 and $504 \mathrm{~nm}$, respectively. This is characteristic to protonation of the carbonyl group and formation of monocation II. In addition, blue shifted bands appear at 259, 257 and $258 \mathrm{~nm}$, respectively, characteristic for protonation of $-\mathrm{NMe}_{2}$ and the carbonyl group; and formation of dications (scheme 3). These changes are also accompanied by appearance of sharp isosbestic points at 469, 448 and $423 \mathrm{~nm}$, for DPACP, DPACH and DPAE, respectively. By examining absorption spectra of the investigated dyes in weak organic acid such as trifluoroacetic acid (at 90\%), one absorption band at 415, 397 and $373 \mathrm{~nm}$ for DPACP, DPACH and DPAE has been formed, confirming the protonation of the $-\mathrm{NMe}_{2}$ group and formation of monocation I (see figure 6). No new absorption band at longer wavelength has been observed which is characteristic to the unprotonated form of the carbonyl group and prevents the possibility of monocation II formation, so, the protonation of carbonyl group occurs at very higher concentration of sulphuric acid.

Fluorescence spectra of DPACP, DPACH and DPAE recorded at different concentrations of sulphuric acid by excitation at 450, 430 and $420 \mathrm{~nm}$ for the dyes, respectively. Fluorescence maximum of the neutral species which appear at $\lambda_{\max }^{f}=587,601$ and $574 \mathrm{~nm}$, respectively, are blue-shifted $\left(\lambda_{\max }^{f}=518,514\right.$ and $567 \mathrm{~nm}$, respectively), at lower sulphuric acid concentrations, while they are red-shifted $\left(\lambda_{\max }^{f}=621,618\right.$ and $593 \mathrm{~nm}$, respectively) at higher concentrations of sulphuric acid with appearance of new hypsochromic shifted band at

Table 5. Spectral data, ground $\left(\mathrm{pK}_{\mathrm{a}}\right)$ and excited $\left(\mathrm{p} K_{\mathrm{a}}^{*}\right)$ protonation constants of the investigated ketocyanines.

\begin{tabular}{|c|c|c|c|c|c|c|c|c|c|c|c|c|}
\hline \multirow[b]{2}{*}{ Dye } & \multicolumn{2}{|c|}{ Neutral form } & \multicolumn{2}{|c|}{ Monocation I } & \multicolumn{2}{|c|}{ Monoction II } & \multicolumn{2}{|c|}{ Dication } & \multirow[b]{2}{*}{$\lambda_{i s o}(\mathrm{~nm})$} & \multicolumn{2}{|c|}{$\mathrm{p} K_{\mathrm{a}}$} & \multirow{2}{*}{$\begin{array}{c}\mathrm{p} K_{\mathrm{a}}^{*} \\
\mathrm{FC}\end{array}$} \\
\hline & $\lambda_{a}(\mathrm{~nm})$ & $\lambda_{f}(\mathrm{~nm})$ & $\lambda_{a}(\mathrm{~nm})$ & $\lambda_{f}(\mathrm{~nm})$ & $\lambda_{a}(\mathrm{~nm})$ & $\lambda_{f}(\mathrm{~nm})$ & $\lambda_{a}(\mathrm{~nm})$ & $\lambda_{f}(\mathrm{~nm})$ & & HHM & LAM & \\
\hline DPACP & 427 & 587 & 415 & 518 & 574 & 621 & 259 & 494 & 469 & -3.74 & -3.68 & $\begin{array}{l}2.97^{(\mathrm{a})} \\
3.06^{(\mathrm{b})}\end{array}$ \\
\hline DPACH & 414 & 601 & 397 & 514 & 554 & 618 & 257 & 488 & 448 & -4.08 & -3.86 & $\begin{array}{c}3.15^{(\mathrm{a})} \\
3.2^{\text {(b) }}\end{array}$ \\
\hline DPAE & 409 & 574 & 373 & 567 & 504 & 593 & 258 & 484 & 423 & -4.3 & -4.21 & $\begin{array}{l}1.08^{(\mathrm{a})} \\
1.31^{(\mathrm{b})}\end{array}$ \\
\hline
\end{tabular}

(a) Calculated using the $\mathrm{p} K_{\mathrm{a}}$ values obtained from HHM

(b) Calculated using the $\mathrm{p} K_{\mathrm{a}}$ values obtained from LAM 


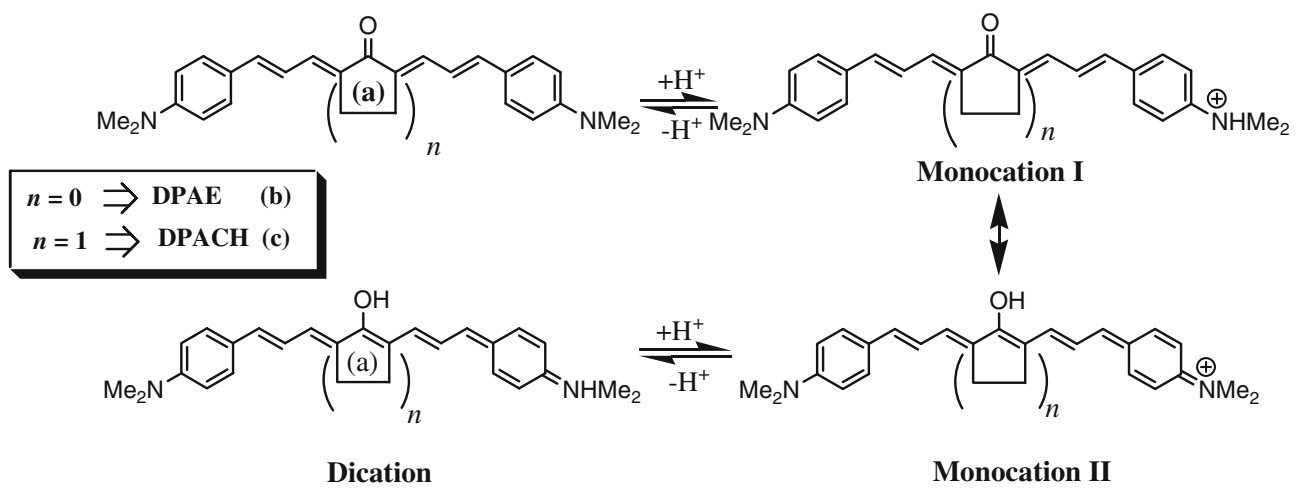

Scheme 3. Prototropic equilibria of (a) DPACP, (b) DPACH and (c) DPAE.

494, 488 and $484 \mathrm{~nm}$, respectively. These results can be explained on the basis of the ICT character of the lowest energy transition as well as the structure of the investigated ketocyanines as follows: the molecule has two protonation sites, one is the nitrogen atom of the dimethylamino group and the other is the oxygen atom of carbonyl group. So, it is expected that addition of a proton to oxygen atom of the carbonyl group will shift the absorption and fluorescence maxima to red due to enhancement of charge-transfer from the dimethylamino group to carbonyl group. In contrast, protonation of the $-\mathrm{NMe}_{2}$ group shifts the spectra to blue compared to the neutral molecules. Thus, it could be concluded that the two bands in the absorption spectrum are due to two different species which are also present in the excited state. These species, as shown in scheme 3, are the monocations I and II. The species

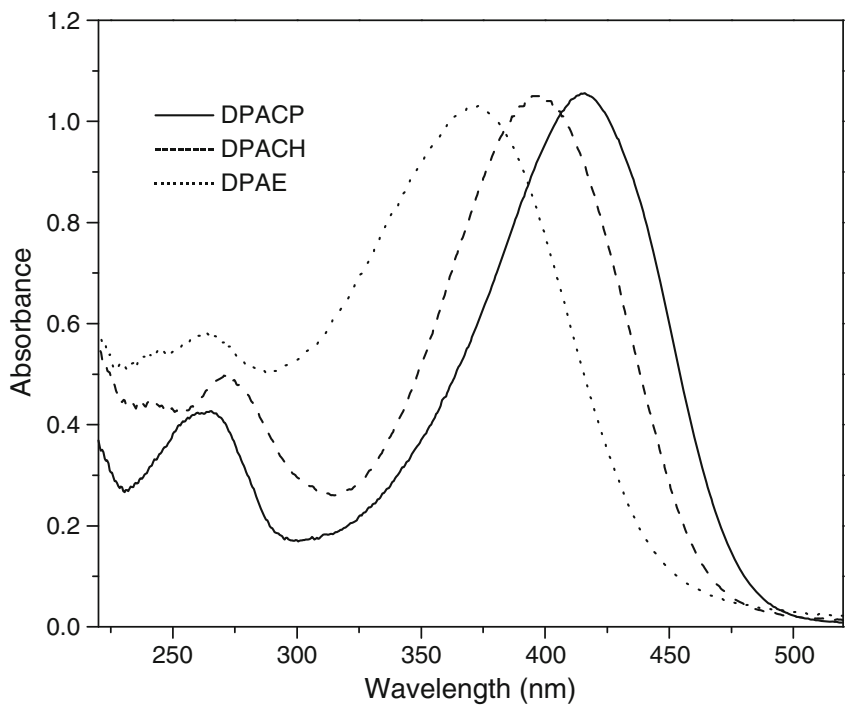

Figure 6. Absorption spectra of DPACP (-), DPACH (- - ) and DPAE (.....) in trifluoroacetic acid. responsible for the blue-shifted absorption and emission spectra compared to those of monocation I and redshifted compared to those of monocation II is assigned to the dication.

Protonation constants of the investigated ketocyanines in the ground state have been determined by two methods, half-height method (HHM) ${ }^{22}$ and limiting absorbance methods (LAM) ${ }^{23}$ From the inflection point of the spectrophotometric titration curves, the ground state $\mathrm{p} K_{\mathrm{a}}$-values were determined and listed in table 5 together with spectral maxima of different species. Protonation constants of the investigated ketocyanines in the first singlet excited state $\left(\mathrm{p} K_{\mathrm{a}}^{*}\right)$ are determined by using Forster cycle. ${ }^{24}$ The values summarized in table 5 show that the $\mathrm{p} K_{\mathrm{a}}^{*}$ values are lower than $\mathrm{p} K_{\mathrm{a}}$ values calculated for the ground state. This indicates that the investigated ketocyanines become more basic in the first excited singlet state than in the ground state.

\section{Conclusion}

Some symmetrical (D- $\pi-A-\pi-D)$ ketocyanines have been synthesized and their solvato- and acidochromic properties have been studied. Their spectral characteristics suggest that they can serve as good reporters of solvent polarity, hydrogen-bond donating ability and the acidity of the medium (in other words, the water content of concentrated sulphuric acid). In comparison with the corresponding unsymmetrical dye (DMAPT), the absorption and fluorescence maxima of the investigated symmetrical dyes are very sensitive to hydrogen bond donation by solvent relative to DMAPT, while the $\Phi_{f}$ values for symmetric DPACP, DPACH and DPAE are much lower than that for the corresponding unsymmetrical DMAPT, indicating a slower decay of the emitting state for symmetric dye. Dramatic shifts in the 
absorption and emission spectra as well as the observed change in colour upon increasing the concentrations of sulphuric acid lead to design of potential optical sensors for probing acidity of the medium. The results have been confirmed with theoretical calculations using PM3 and ZINDO/S semiempirical methods.

\section{References}

1. Bhattacharya K and Chowdhury M 1993 Chem. Rev. 93 507

2. Reichardt C 1994 Chem. Rev. 942319

3. Lakowicz J R 1983 Principles of fluorescence spectroscopy (New York: Plenum)

4. Etaiw H S, Fayed A T and Saleh Z N 2006 J. Photochem. Photobiol. A Chem. 177238

5. Tada B E, Silva L P and El Seoud A O 2003 J. Phys. Org. Chem. 161

6. Gastilovich A E, Korol'kova V N, Val'kova A G, Konoplev G G and Ni V B 1992 Russ. J. Phys. Chem. 66 1121

7. Dubois E J, Goetz E and Bienvenue A 1964 Spectrochim. Acta A24 1815

8. Reichardt C, Asharin-Fard S and Schafer G 1993 Liebigs Ann. Chem. 123
9. Balamurali M M and Dogra K S 2002 J. Photochem. Photobiol. A Chem. 15481

10. Connors E R and Ucak-Astarlioglu G M 2003 J. Phys. Chem. A107 7684

11. Rochester H C 1970 Acidity functions (London, New York: Academic Press)

12. Thompson A M, Glendening D E and Feller D $1994 \mathrm{~J}$. Phys. Chem. 9810465

13. Fayed A T, El-morsi A M and El-Nahass N M 2011 J. Photochem Photobiol. A Chem. 22438

14. Maiti N C, Mazumdar S and Periasamy N 1998 J. Phys. Chem. B102 1528

15. Sapagovas V J, Gaidelis V, Kovalevskij V and Undzenas A 2006 Dyes Pigments $\mathbf{7 1} 178$

16. Thiagarajan V, Selvaraju C, Padmamalar J E and Ramamurthy P 2004 Chem. Phys. Chem. 51200

17. Wang Y 1985 J. Phys. Chem. 893799

18. Bakhshiev G N 1964 Opt. Spectrosc. 16821

19. Chamma A and Viallet P 1970 Determination of the dipole moment in a molecule in an excited singlet state. C.R. Acad. Sci. Paris Ser C. 270

20. Kawasaki A 2002 Z. Naturforsch. A57 255

21. Hermant M R, Bakker C A N, Scherer T, Krijinen B and Verboeven W J 1990 J. Am. Chem. Soc. 1121214

22. Issa M R 1971 J. Chem. 14133

23. Ibrahim A N, Issa M R, Zayan E S and El-Hefnawey B G 1973 J. Prakt. Chem. 315202

24. Forster T Z 1950 Phys. Chem. 5443 